\title{
ONE-TO-ONE MAPPINGS OF RINGS AND LATTICES
}

\author{
C. E. RICKART
}

1. Introduction. Let $R$ and $S$ be two arbitrary rings and let $\phi(r)$ denote a one-to-one, multiplicative mapping of $R$ onto $S$. The major portion of this paper is devoted to the proofs of two theorems which state that, under certain conditions on $R$, the mapping $\phi(r)$ is automatically additive. In Theorem I ( $\$ 2), R$ is an arbitrary Boolean ring and in Theorem II ( $\$ 3), \mathcal{R}$ is any ring which contains a family $\mathcal{F}$ of minimal right ideals satisfying the following two conditions: (i) $R r=(0)$, for every $R \in \mathcal{F}$, implies $r=0$ and (ii) each $R \in \mathcal{F}$ is of dimension greater than one over the division ring of all endomorphisms of $R$ which commute with each endomorphism induced in $R$ through right multiplication by elements of $R$. In $\$ 4$ it is proved (Theorem III) that a one-to-one, meet preserving mapping of a distributive lattice onto a distributive lattice is necessarily join preserving.

The class of rings considered in Theorem II contains as a special case the ring of all bounded linear operators on a Banach space of dimension greater than one. Theorem II, with the additional hypothesis that the mapping be continuous, was proved in this special case by Eidelheit ${ }^{1}[2] .^{2}$ The finite-dimensional case of the Eidelheit theorem was obtained by Nagumo [5].

This paper has been greatly influenced by conversations which we have had with B. J. Pettis. In particular, our interest in the questions considered here was stimulated by Pettis' conjecture that the Eidelheit theorem mentioned above could be obtained without the continuity hypothesis.

2. Boolean rings. By a Boolean ring [6] we mean a ring each of whose elements satisfies the conditions $r^{2}=r$. It is not difficult to show that a Boolean ring is necessarily commutative and that each of its elements also satisfies the condition $r+r=0$.

THEOREM I. Any one-to-one, multiplicative mapping of a Boolean ring $\mathcal{B}$ onto an arbitrary ring $\mathcal{S}$ is necessarily additive.

Let $\phi(r)$ denote the one-to-one, multiplicative mapping of $\mathbb{B}$ onto

Presented to the Society, October 25, 1947; received by the editors September 20, 1947.

${ }^{1}$ Eidelheit, in fact, assumed $S$, as well as $R$, to consist of all bounded linear operators on a Banach space. He also assumed the Banach spaces to be real.

2 Numbers in brackets refer to the bibliography at the end of the paper. 
S. It is evident that the multiplicative condition on $\phi(r)$ implies that $\delta$ must also be a Boolean ring. Moreover $\phi(r)=0$ if, and only if, $r=0$. In fact, since the range of $\phi(r)$ covers $S$, there exists $u \in \mathcal{B}$ such that $\phi(u)=0$. Therefore

$$
\phi(0)=\phi(0 u)=\phi(0) \cdot \phi(u)=0 .
$$

That $u=0$ follows from the fact that $\phi(r)$ is one-to-one.

Now let $r, s$ be arbitrary elements of $\mathcal{B}$ and choose $t \in \mathcal{B}$ so that

$$
\phi(t)=\phi(r)+\phi(s) .
$$

Observe that, since the mapping is multiplicative,

$$
\begin{aligned}
\phi(r t+s t) & =\phi(r+r s)+\phi(s+r s), \\
\phi(r t) & =\phi(r)+\phi(r s), \\
\phi(s t) & =\phi(r s)+\phi(s) .
\end{aligned}
$$

Consider first the case in which $r s=0$. Then (1), (2) and (3) become

$$
\begin{aligned}
\phi(r t+s t) & =\phi(r)+\phi(s), \\
\phi(r t) & =\phi(r), \\
\phi(s t) & =\phi(s) .
\end{aligned}
$$

Since the mapping is one-to-one, equations $(1)^{\prime},(2)^{\prime}$ and (3)' imply respectively $r t+s t=t, r t=r$ and $s t=s$. It follows that $t=r+s$ and therefore

$$
\phi(r+s)=\phi(r)+\phi(s) .
$$

Now consider the case in which $r s=s$. Then (1), (2) and (3) become

$$
\begin{aligned}
\phi(r t+s t) & =\phi(r+s), \\
\phi(r t) & =\phi(r)+\phi(s), \\
\phi(s t) & =0 .
\end{aligned}
$$$$
(3)^{\prime \prime}
$$

Again, since the mapping is one-to-one, equations (1)", (2)" and (3) ${ }^{\prime \prime}$ imply respectively $r t+s t=r+s, r t=t$ and $s t=0$. Therefore, $t=r+s$ and it follows that equation (4) holds in this case as well.

In the general case, observe that

$$
r+s=(r+r s)+(s+r s) .
$$

Since $(r+r s)(s+r s)=0$, the first case considered above gives

$$
\phi(r+s)=\phi(r+r s)+\phi(s+r s) .
$$


Moreover, since $r(r s)=r s$ and $s(r s)=r s$, the second case considered above gives

$$
\phi(r+r s)=\phi(r)+\phi(r s), \phi(s+r s)=\phi(s)+\phi(r s) .
$$

Substituting these values in (5), we again obtain (4) which completes the proof.

COROLLARy. In a Boolean ring, the operation of addition is uniquely determined by the operation of multiplication.

3. Rings with minimal ideals. Let $R$ be an arbitrary ring which contains a minimal right ideal $R$. For each $r \in \mathcal{R}$, let $T_{r}$ denote the endomorphism of the additive group of $R$ defined by $x T_{r}=x r, x \in R$. Since $R$ is minimal, the $T_{r}$ constitute an irreducible representation of $R$ in the ring of endomorphisms of $R$. By Schur's lemma, the family $\Phi$ of all endomorphisms of $R$ which commute with each $T_{r}$ is a division ring. Therefore, $R$ can be considered as a linear vector space over $\Phi$. The linear space associated in this way with $R$ will be denoted by $\bar{R}$. By a result of Jacobson [3, Theorem 6], the $T_{r}$ constitute a dense ring of linear operators on $\bar{R}$. In other words, for arbitrary positive integer $k$ and arbitrary elements $x_{1}, \cdots, x_{k}, y_{1}, \cdots, y_{k}$ in $R$, where the $x_{1}, \cdots, x_{k}$ are linearly independent in $\bar{R}$, there exists $r \in \mathbb{R}$ such that $x_{i} r=y_{i}$ for $i=1, \cdots, k$.

LEMMA. Let $R$ be a ring which contains a minimal right ideal $R$ whose associated linear vector space $\bar{R}$ is of dimension greater than one. Then any one-to-one, multiplicative mapping of $\mathbb{R}$ onto an arbitrary ring $\mathcal{S}$ is additive on $R$.

Let $\phi(r)$ denote the one-to-one, multiplicative mapping of $R$ onto $\delta$ and observe (as in the proof of Theorem I) that $\phi(r)=0$ if, and only if, $r=0$.

Consider arbitrary elements $x, y \in R$ and choose $z \in R$ such that

$$
\phi(z)=\phi(x)+\phi(y) .
$$

Assume first that $x, y$ are linearly independent in $\bar{R}$. Then, by the density property, there exist $r, s \in \mathcal{R}$ such that

$$
x r=x, \quad y r=0, \quad x s=0, \quad y s=y .
$$

Observe that

$$
\begin{aligned}
\phi(z r) & =\phi(x) \cdot \phi(r)+\phi(y) \cdot \phi(r) \\
& =\phi(x r)+\phi(y r) \\
& =\phi(x) .
\end{aligned}
$$


Therefore, since the mapping is one-to-one, $z r=x$. Similarly we obtain $z s=y$. It follows that

$$
x+y=z(r+s) .
$$

Note also that

$$
x(r+s)=x, \quad y(r+s)=y .
$$

Therefore

$$
\begin{aligned}
\phi(x+y) & =\phi(z(r+s)) \\
& =\phi(x) \cdot \phi(r+s)+\phi(y) \cdot \phi(r+s) \\
& =\phi(x(r+s))+\phi(y(r+s)) \\
& =\phi(x)+\phi(y) .
\end{aligned}
$$

This proves that $\phi(r)$ is additive on linearly independent elements of $\bar{R}$.

Next assume that $x$ and $y$ are linearly dependent in $\bar{R}$. Since $\bar{R}$ is of dimension greater than one, there exists $u \in \bar{R}$ linearly independent of $x$ and $y$. Since $\phi(r)$ is additive on linearly independent elements of $\bar{R}$, we have

$$
\begin{aligned}
\phi(x+y)+\phi(u) & =\phi(x+y+u) \\
& =\phi(x)+\phi(y+u) \\
& =\phi(x)+\phi(y)+\phi(u) .
\end{aligned}
$$

Therefore

$$
\phi(x+y)=\phi(x)+\phi(y)
$$

and the proof is complete.

COROLlaRy. Under the hypothesis of the lemma, $\phi(R)$ is a minimal right ideal in $\mathrm{S}$.

THEOREM II. Let $R$ contain a family $\mathcal{F}$ of minimal right ideals which satisfy the following conditions: (i) $R r=(0)$, for each $R \in \mathcal{F}$, implies $r=0$; (ii) The linear space $\bar{R}$ associated with each $R \in \mathcal{F}$ is of dimension greater than one. Then any one-to-one, multiplicative mapping of $R$ onto an arbitrary ring $\mathcal{S}$ is necessarily additive.

Denote by $\phi(r)$ the one-to-one, multiplicative mapping of $R$ onto $S$. Let $r, s$ be arbitrary elements of $R$ and choose $t \in R$ such that

$$
\phi(t)=\phi(r)+\phi(s) .
$$

The proof consists in showing that $t=r+s$. Let $R$ be any element 
of $\mathcal{F}$ and observe that, if $x \in R$, then

$$
\phi(x t)=\phi(x r)+\phi(x s) .
$$

Moreover, by the above lemma,

$$
\phi(x r+x s)=\phi(x r)+\phi(x s) .
$$

Therefore

$$
x r+x s=x t
$$

It follows that

$$
R(r+s-t)=(0) .
$$

Since $R$ was an arbitrary element of $\mathcal{F}$, we conclude that $t=r+s$.

COROLlARY. In any ring which satisfies the conditions of Theorem II, the operation of addition is uniquely determined by the operation of multiplication.

COROLlary. Under the hypothesis of Theorem II, any one-to-one, anti-multiplicative $e^{3}$ mapping of $R$ onto an arbitrary ring is necessarily additive.

The following example shows that the dimension restriction in Theorem II cannot be dropped. It also shows, incidentally, that the Boolean ring in Theorem I cannot be replaced by a generalized Boolean ring ${ }^{4}$ in the sense of McCoy and Montgomery [4]. Let $R$ and $S$ both equal the finite field $F_{p}$ of integers modulo the prime $p$. In this case, $R$ is a generalized Boolean ring (of index $p$ ) which contains only one minimal ideal, namely, $R$ itself. Condition (i) of Theorem II is clearly satisfied, although the dimension condition (ii) does not hold. Now consider the mapping $\phi(x)$ of $R$ into $S$ defined by $\phi(x)=x^{3}$. It is obvious that $\phi(x)$ is multiplicative. Moreover, in the special case $p=5, \phi(x)$ defines a one-to-one mapping of $R$ onto $S$. In fact, $\phi(0)=0$, $\phi(1)=1, \phi(2)=3, \phi(3)=2$ and $\phi(4)=4$. However, since $\phi(1+2)$ $\neq \phi(1)+\phi(2)$, the mapping is not additive. The above example, with $R$ and $S$ equal to the field of real numbers instead of $F_{5}$, was given by Eidelheit [2, p. $103 \mathrm{nb}]$.

It is perhaps desirable to remark briefly on the relationship between Theorems I and II. Note, in the first place, that in Theorem I the Boolean ring $\mathcal{B}$ need not contain any minimal ideals. Moreover, even

${ }^{3}$ That is, $\phi(r s)=\phi(s) \phi(r)$.

4 A generalized Boolean ring (of index $p$ ) is a commutative ring for which there exists a prime $p$ such that, for every element $r$ of the ring, $r^{p}=r$ and $p r=0$. 
if $\mathcal{B}$ does contain a minimal ideal $R$, it is evident that $R$ contains only one nonzero element. It follows that $\bar{R}$ cannot have dimension greater than one. Therefore, the hypotheses of Theorem II cannot be satisfied for a Boolean ring. On the other hand, it is trivial to verify that the lemma for Theorem II holds for Boolean rings without the dimension requirement. Hence, the proof of Theorem II could still be carried through for Boolean rings if condition (i) were satisfied. We also note at this point that Theorem I could be obtained from Theorem III of the next section.

4. Distributive lattices. We obtain now a theorem for distributive lattices $^{5}$ analogous to the ring theorems obtained above.

TheOREM III. Any one-to-one, meet-preserving mapping of a distributive lattice onto a distributive lattice is also join-preserving.

Let $\mathcal{L}$ and $\mathscr{X}$ be arbitrary distributive lattices and let $\phi(x)$ denote a one-to-one, meet-preserving mapping of $\mathcal{L}$ onto $\mathcal{X}$. Consider two arbitrary elements $x, y \in \mathcal{L}$ and choose $z \in \mathcal{L}$, such that

$$
\phi(z)=\phi(x) \cup \phi(y) .
$$

The proof consists in showing that $z=x \cup y$. Observe first that

$$
\begin{aligned}
\phi(z \cap(x \cup y)) & =\phi(z) \cap \phi(x \cup y) \\
& =[\phi(x) \cap \phi(x \cup y)] \cup[\phi(y) \cap \phi(x \cup y)] \\
& =\phi(x \cap(x \cup y)) \cup \phi(y \cap(x \cup y)) \\
& =\phi(x) \cup \phi(y) \\
& =\phi(z) .
\end{aligned}
$$

Since the mapping is one-to-one, it follows that

$$
z=z \cap(x \cup y)=(z \cap x) \cup(z \cap y) .
$$

Also

$$
\phi(z \cap x)=[\phi(x) \cap \phi(x)] \cup[\phi(y) \cap \phi(x)]=\phi(x) \cup \phi(y \cap x) .
$$

But

$$
\phi(x) \cap \phi(y \cap x)=\phi(x \cap y \cap x)=\phi(y \cap x) .
$$

Therefore

$$
\phi(z \cap x)=\phi(x) \cup \phi(y \cap x)=\phi(x) .
$$

Similarly

5 The terminology used here conforms with [1]. 


$$
\phi(z \cap y)=\phi(y) .
$$

Again, since the mapping is one-to-one, we have $z \cap x=x$ and $z \cap y=y$. It follows that $z=x \cup y$ and the proof is complete.

Since the dual of a distributive lattice is also distributive, we can state the following corollaries:

Corollary. Any one-to-one, join-preserving mapping of a distributive lattice onto a distributive lattice is also meet-preserving.

Corollary. Any one-to-one mapping of a distributive lattice onto a distributive lattice which takes meets into joins also takes joins into meets.

\section{BIBLIOGRAPHY}

1. Garrett Birkhoff, Lcttice theory, Amer. Math. Soc. Colloquium Publications, vol. 25, New York, 1940.

2. M. Eidelheit, On isomorphisms of rings of linear operators, Studia Math. vol. 9 (1940) pp. 97-105.

3. N. Jacobson, Structure theory of simple rings without finiteness assumptions, Trans. Amer. Math. Soc. vol. 57 (1945) pp. 228-245.

4. N. H. McCoy and Deane Montgomery, A representation of generalized Boolean rings, Duke Math. J. vol. 3 (1937) pp. 455-459.

5. M. Nagumo, Über eine kennzeichnende Eigenshaft der Linearkombinationen von Vektoren und ihre Anwendung, Nachr. Ges. Wis. Göttingen (1933) pp. 36-40.

6. M. H. Stone, The theory of representations for Boolean algebras, Trans. Amer. Math. Soc. vol. 40 (1936) pp. 37-111.

YALE UNIVERSITY 